\title{
Pleural irrigation with normal saline for empyema
}

Pleural infection remains a serious condition that has significant morbidity and mortality. ${ }^{[1]}$ In wellresourced countries the mortality is estimated to be approximately $20 \%$ in the 6 months following the initial presentation. ${ }^{[2]}$ Despite this alarming mortality, controversy remains regarding the management and specifically the role of fibrinolytic therapy ${ }^{[3]}$ The impact of pleural infections is likely to be greater in resource-limited countries like South Africa where there is a high prevalence of infectious diseases. Medical management with intravenous antibiotics and intercostal drainage is the mainstay of treatment, but this fails in a proportion of patients, mandating surgical intervention. Pre-emptive surgical management is of unproven benefit and may be costly. In 2011, The Multi-centre Intra-pleural Sepsis Trial (MIST 2) suggested that a combination of tissue plasminogen activator and DNase improves the chest radiographic appearance after a week, but this approach is expensive and requires further validation. ${ }^{[4]}$

Intrapleural saline irrigation is a simple technique that has been performed in many European countries despite the lack of evidence of its efficacy from controlled trials. A recent randomised controlled pilot study - the Pleural Irrigation Trial (PIT) - was performed in 35 patients with pleural infection requiring chest-tube drainage in which pleural irrigation with saline plus best-practice management was compared with the latter alone. ${ }^{[5]}$ Patients receiving the additional saline irrigation had a greater reduction in pleural collection volume on computed tomography compared with those receiving standard care: $32.3 \%$ v. $15.3 \%$. Additionally, significantly fewer patients in the irrigation group were subsequently referred for surgery (odds ratio 7.1,
$95 \%$ confidence interval $1.23-41.0 ; p=0.03$ ). There was no difference in length of hospital stay, fall in C-reactive protein, white cell count, procalcitonin or adverse events between the treatment groups, and no serious complications were documented. This pilot study indicates that pleural irrigation with normal saline may increase pleural fluid drainage and reduce referrals for surgery in pleural infections. This may provide a simple and cost-effective alternative to more expensive regimens should the results of this pilot study be reproduced in larger controlled studies.

\section{Dr Donald Simon}

Pulmonology Fellow, Division of Pulmonology, Department of Medicine, Stellenbosch University, South Africa

\section{References}

1. Chapman SJ, Davies RJ. Recent advances in parapneumonic effusions and empyema. Curr Opin Pulm Med 2004;10(4):299-304

2. Davies CW, Kearney SE, Gleeson FV, Davies RJ. Predictors of outcome and longterm survival in patients with pleural infection. Am J Respir Crit Care Med 1999;160(5):1682-1687. [http://dx.doi.org/10.1164/ajrccm.160.5.9903002]

3. Koegelenberg CFN, Diacon AH, Bolliger CT. Parapneumonic pleural effusion and empyema. Respiration 2008;75(3):241-250. [http://dx.doi.org/10.1159/000117172]

4. Rahman NM, Maskell NA, West A, et al. Intrapleural use of tissue plasminogen activator and DNase in pleural infection. N Engl J Med 2011;365(6):518-526. [http:// dx.doi.org/10.1056/NEJMoa1012740]

5. Hooper CE, Edey AJ, Wallis A, et al. Pleural irrigation trial (PIT): A randomised controlled trial of pleural irrigation with normal saline versus standard care in patients with pleural infection. Eur Respir J 2015;46(2):456-463. [http://dx.doi. org/10.1183/09031936.00147214]

\section{Three rapid tests for the diagnosis of drug-resistant tuberculosis}

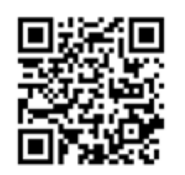

Multidrug-resistant tuberculosis (MDR-TB) is more difficult and costly to treat than drug-susceptible TB and patient outcomes are unfortunately worse. In South Africa, detection of drug-resistant tuberculosis (DR$\mathrm{TB})$ increases each year, ${ }^{[1]}$ and curing and preventing the spread of these strains of TB bacteria requires a wide range of resources, including prompt diagnosis and effective antibiotics. One such antibiotic, Bedaquiline, ${ }^{[2]}$ is the first drug in a new class of antiTB medications to be approved in more than 40 years by the US Food and Drug Administration (FDA). More effective treatment strategies mean that diagnosing MDR-TB becomes even more important in combating this increasing global burden.

In a recent multicentre, prospective, head-to-head clinical evaluation study by Catanzaro et al., ${ }^{[3]}$ the performance of three rapid tests - two DNA-based rapid diagnostic assays (line probe assay and pyrosequencing) and one growth-based assay - were evaluated for diagnosing drug resistance in patients at risk for $\mathrm{M} / \mathrm{DR}-\mathrm{TB}$, relative to the World Health Organization's approved phenotypic reference standard of the Mycobacterium growth indicator tube and drug susceptibility testing (MGIT DST). Sputum from 1128 study participants at TB clinics in India, Moldova and South Africa were examined. The comparisons showed that all three rapid assays accurately identified resistance to first- and second-line oral antibiotic treatments (isoniazid, rifampin, moxifloxacin and ofloxacin). They were less accurate but still very good at detecting resistance to the injectable antibiotics (amikacin and capreomycin) but performed poorly in detecting resistance to only one drug, kanamycin. As expected, the molecular techniques were superior when comparing the time it took to obtain results, with a mean time of 1.1 days for both DNA testing methods, 14.3 days for the rapid culture method, and 24.7 days for the reference standard test.

Although the two molecular techniques may be costly, the third technique employs a low-cost and easy-to-use version of the standard bacterial culture technique, making it suitable for resource-limited community clinics and hospitals. It would seem that the World Health Organization's goal of reducing deaths due to TB by $95 \%$ by 2050 may be achievable with such developments in improved diagnostics.

\section{Dr Morné Vorster}

Pulmonology Fellow, Division of Pulmonology, Department of Medicine, Stellenbosch University, South Africa 


\section{References}

1. Hughes J, Osman M. Diagnosis and management of drug-resistant tuberculosis in South African adults. S Afr Med J 2014;104(12):894.

2. Diacon AH, Pym A, Grobusch MP, et al. Multidrug-resistant tuberculosis and culture conversion with Bedaquiline. N Engl J Med 2014;371(8):723-732. [http://dx.doi. org/10.1056/NEJMoa1313865
3. Catanzaro A, Rodwell TC, Catanzaro DG, et al. Performance comparison of three rapid tests for the diagnosis of drug-resistant tuberculosis. PLoS One 2015;10(8):e0136861. [http://dx.doi.org/10.1371/journal.pone.0136861]

\section{Lung-function trajectories leading to chronic obstructive pulmonary disease}

Chronic obstructive pulmonary disease (COPD) is the third leading cause of morbidity and mortality worldwide. A gradual decline in forced expiratory volume in 1 second $\left(\mathrm{FEV}_{1}\right)$ is a normal part of ageing. After maximal lung function is obtained, lung function as remains steady with very minimal change from age 20 to 35 years and thereafter starts declining. However, this rate of decline is accelerated in patients with COPD and continued smokers, ${ }^{[1]}$ with greater decline in the early stages of COPD (GOLD II and III), ${ }^{[2]}$ emphasising the importance of early smoking cessation interventions.

However, accelerated decline is not the only trajectory for the development of COPD. Lange et al. ${ }^{[3]}$ recently reported that accelerated decline is not obligate in the genesis of COPD and low $\mathrm{FEV}_{1}$ attained during young adulthood increases the risk of developing COPD. This observation stemmed from a retrospective study involving three large cohorts - the Framingham Offspring Cohort, the Copenhagen City Heart Study, and the Lovelace Smokers Cohort. The FEV 1 ( $\geq 80 \%$ or $<80 \%$ of the predicted value) at cohort inception (before 40 years old) as well as at the last cohort visit were measured. The rate of decline in the $\mathrm{FEV}_{1}$ over time according to their $\mathrm{FEV}_{1}$ at cohort start and COPD status (GOLD grade 2/higher) at final visit was determined.

There were 4417 subjects and 495 had COPD at cohort end. Among the 657 participants who had an $\mathrm{FEV}_{1}$ of less than $80 \%$ of the predicted value before 40 years of age, 174 (26\%) had COPD, whereas among 2207 subjects who had a baseline $\mathrm{FEV}_{1}$ of at least $80 \%$ of the predicted value before 40 years of age, 158 (7\%) had COPD. Approximately half the persons with COPD had a normal $\mathrm{FEV}_{1}$ before 40 years of age and had a rapid decline in $\mathrm{FEV}_{1}$ thereafter, while the remaining half had a low $\mathrm{FEV}_{1}$ during early adulthood with a normal decline thereafter.

This study suggests that a low lung function or $\mathrm{FEV}_{1}$ with normal rate of decline during early adulthood is an important risk factor for the development of COPD and that accelerated decline in lung function from a normal $\mathrm{FEV}_{1}$ is not a prerequisite for the genesis of COPD.

\section{Dr Shinu Abraham}

Discovery Sub-specialist Fellow, Division of Pulmonology, Department of Medicine, Stellenbosch University, South Africa

\section{References}

1. O'Hara P, Grill J, Rigdon MA, Connett JE, Lauger GA, Johnston JJ. Design and results of the initial intervention program for the Lung Health Study. Prev Med 1993;22(3):304-315

2. Tantucci C, Modina D. Lung function decline in COPD. Int J Chron Obstruct Pulmon Dis 2012;7:95-99. [http://dx.doi.org/10.2147/COPD.S27480]

3. Lange P, Celli B, Agustí A, et al. Lung-function trajectories leading to chronic obstructive pulmonary disease. N Engl J Med 2015;373(2):111-122. [http://dx.doi. org/10.1056/NEJMoal411532]

S Afr Resp J 2015;21(3):82-83. DOI:10.7196/10.2015.v21i3.83 